\title{
Leptin surge precedes onset of autoimmune encephalomyelitis and correlates with development of pathogenic $T$ cell responses
}

\author{
Veronica Sanna, ${ }^{1}$ Antonio Di Giacomo, ${ }^{2}$ Antonio La Cava, ${ }^{3}$ Robert I. Lechler, ${ }^{4}$ \\ Silvia Fontana, ${ }^{1}$ Serafino Zappacosta, ${ }^{1}$ and Giuseppe Matarese ${ }^{1}$
}

\begin{abstract}
${ }^{1}$ Gruppo di ImmunoEndocrinologia, Istituto di Endocrinologia e Oncologia Sperimentale, Consiglio Nazionale delle Ricerche (IEOS-CNR) and Cattedra di Immunologia, Dipartimento di Biologia e Patologia Cellulare e Molecolare, Università di Napoli "Federico II," Napoli, Italy

${ }^{2}$ Laboratorio di Immunologia Cellulare, Azienda Ospedaliara "V. Monaldi," Napoli, Italy

${ }^{3}$ Autoimmunity and Tolerance Laboratory, Department of Medicine, University of California, Los Angeles, California, USA

${ }^{4}$ Department of Immunology, Imperial College School of Medicine, Hammersmith Hospital, London, United Kingdom
\end{abstract}

In the work presented here, we explored the influence of leptin on the kinetics of experimental autoimmune encephalomyelitis (EAE) onset, in the EAE-associated inflammatory anorexia, and in the development of pathogenic $\mathrm{T}$ cell responses. We found that the expression of serum leptin increased before the clinical onset of EAE in disease-susceptible C57BL/6J $\left(\mathrm{H}-2^{\mathrm{b}}\right)$ and SJL/J $\left(\mathrm{H}-2^{\mathrm{s}}\right)$ strains of mice, which are models of chronic-progressive and relapsing-remitting EAE, respectively. This increase in serum leptin correlated with disease susceptibility, reduction in food intake, and decrease in body weight. Indeed, acute starvation, which is able to prevent the increase in serum leptin, delayed disease onset and attenuated clinical symptoms by inducing a $T$ helper 2 cytokine switch. Furthermore, immunohistochemical analysis revealed a parallel in situ production of leptin in inflammatory infiltrates and in neurons only during the acute/active phase of both chronic-progressive and relapsing-remitting EAE. We also found that leptin secretion by activated $T$ cells sustained their proliferation in an autocrine loop, since antileptin receptor antibodies were able to inhibit the proliferative response of autoreactive $T$ cells in vitro. Given that leptin appears to regulate EAE susceptibility, inflammatory anorexia, and pathogenic T-cell immune function, we postulate that it may offer a potential target in the treatment of multiple sclerosis.

J. Clin. Invest. 111:241-250 (2003). doi:10.1172/JCI200316721.

\section{Introduction}

Experimental autoimmune encephalomyelitis (EAE) is an animal model of the human demyelinating disease MS $(1,2)$. EAE can be induced in susceptible strains of mice through immunization with self antigens (Ag's) of the CNS such as myelin (1). Two main clinical forms of EAE have been described: the chronic-progressive paralysis in C57BL/6J $\left(\mathrm{H}-2^{\mathrm{b}}\right)$ mice immunized with the myelin oligodendrocyte glycoprotein peptide 35-55 (MOG $35-55)$ and the relapsing-remitting model of EAE in $\mathrm{SJL} / \mathrm{J}\left(\mathrm{H}-2^{\mathrm{s}}\right)$ mice immunized with the proteolipid protein peptide

Received for publication August 22, 2002, and accepted in revised form October 22, 2002.

Address correspondence to: Giuseppe Matarese, Gruppo di ImmunoEndocrinologia, Istituto di Endocrinologia e Oncologia Sperimentale, Consiglio Nazionale delle Ricerche (IEOS-CNR), and Cattedra di Immunologia, Dipartimento di Biologia e Patologia Cellulare e Molecolare, Università di Napoli "Federico II”, via S. Pansini, 5-80131, Napoli, Italy. Phone: 39-081-7463311; Fax:39-081-7463252; E-mail: gmatarese@napoli.com.

Conflict of interest: The authors have declared that no conflict of interest exists.

Nonstandard abbreviations used: experimental autoimmune encephalomyelitis (EAE); antigens (Ag's); 3,3'-diaminobenzidine (DAB); mixed-lymphocyte reactions (MLRs); leptin receptor (ObR); mixed lymphocyte reaction (MLR).
139-151 ( $\left.\mathrm{PLP}_{139-151}\right)(3,4)$. A direct role of CD4 ${ }^{+} \mathrm{T}$ cells in the pathogenesis of EAE has repeatedly been shown in adoptive transfer studies, in which myelin-reactive Th1 $\mathrm{CD}^{+}$cells induce disease after transfer (1). Thus, EAE can be induced by either active immunization with myelin Ag's or by passive transfer of activated myelinspecific $\mathrm{T}$ cells. It has long been known that the clinical course of EAE commences with weight loss followed by an ascending paralysis $(1,5)$. Leptin, a cytokine-like hormone with pleiotropic functions, influences food intake, basal metabolism, hematopoiesis, inflammation, and immunity against infectious pathogens (6-8). Recently, it has also been demonstrated that leptin promotes and sustains immune responses mediated by Th1 $\mathrm{CD}^{+} \mathrm{lym}$ phocytes (9). In particular, it has been shown that leptin costimulates naive $\mathrm{T}$-cell proliferation, enhances IFN- $\gamma$ and TNF- $\alpha$ production, and diminishes IL-4 secretion $(9,10)$. Previously, we reported that leptin-deficient C57BL/6J ob/ob mice were resistant to EAE induction and that their myelin-reactive $\mathrm{T}$ cells produced more IL-4 and less IFN- $\gamma$ than did T cells in wild-type controls (11). Recombinant leptin replacement restored their susceptibility as well as their IFN- $\gamma$ secretion (11). Furthermore, we also investigated the role of leptin in the gender difference in EAE susceptibility $(12,13)$. Indeed, 
leptin shows a marked sexual dimorphism in serum concentrations, which are higher in female mice than in male mice with the same body-fat mass (14). Female $\mathrm{SJL} / \mathrm{J}$ mice are susceptible to EAE induction, whereas their male counterparts are not, showing instead an increased Th2 response to myelin and reduced IL-12 production by antigen-presenting cells $(13,15)$. Treatment with recombinant leptin rendered male SJL/J mice susceptible to EAE induction and increased disease susceptibility in female mice, suggesting that leptin is one of the factors accounting for the gender-related susceptibility to EAE in SJL/J mice (16-18).

In the present study, we analyzed the kinetics of leptin secretion during induction of EAE in leptin-deficient C57BL/6J ob/ob mice and C57BL/6J wild-type controls and in SJL/J male and female mice after immunization. These models allowed us to investigate the influence of leptin on susceptibility and resistance to EAE and on inflammatory anorexia and the development of pathogenic T-cell responses.

\section{Methods}

Mice. Female and male SJL/J mice and C57BL/6J wildtype, C57BL/6J ob/ob leptin-deficient, and C57BL/Ks $d b / d b$ leptin receptor-deficient female mice (4 weeks old) were obtained from Charles River Italia (Calco, Italy) and from Harlan Italy (Corezzana, Italy). Experiments were performed under an approved protocol in accordance with the animal use guidelines of the Istituto Superiore di Sanità (Rome, Italy). All mice were age matched for individual experiments and were group housed according to the experimental protocol (two to six mice per standard cage), with a 12-hour light/dark cycle. Paralyzed mice were afforded easier access to food and water to prevent dehydration.

Antigens. In this study, the peptides used were the immunodominant mouse myelin oligodendrocyte glycoprotein $\left(\mathrm{MOG}_{35-55}\right)$ peptide (MEVGWYRSPFSRVVHLYRNGK) for immunization of the C57BL/6J $o b / o b$ mice and their wild-type controls (3), and the proteolipid protein PLP $_{139-151}$ ) peptide (HSLGKWLGHPDKF) for immunization of SJL/J mice (5). Peptides were synthesized by INBIOS (Pozzuoli, Italy); purity was assessed by high-performance liquid chromatography (>97\% pure), and amino acid composition was verified by mass spectrometry. Peptide batches for in vivo and in vitro assays were all from one preparation, initially solubilized in LPS-free saline solution at a concentration of $4 \mathrm{mg} / \mathrm{ml}$ and stored at $-80^{\circ} \mathrm{C}$.

Leptin replacement during starvation. Mouse recombinant leptin (rLeptin) was purchased from R\&D Systems (Minneapolis, Minnesota, USA). Purity was at least 97\%, as determined by SDS-PAGE and silver-staining analyses. Endotoxin content was no more than $0.1 \mathrm{ng} / \mu \mathrm{g}$ of leptin, as determined by the Limulus amebocyte lysate method. For leptin replacement during starvation, a group of female SJL/J mice were starved for 48 hours at priming with PLP ${ }_{139-151}$ and injected intraperitoneally with $200 \mu \mathrm{l}$ of PBS twice daily, whereas another group of mice was injected intraperitoneally twice daily with recombinant leptin dissolved in $200 \mu \mathrm{l}$ of PBS at a dose of $2 \mu \mathrm{g} / \mathrm{g}$ of initial body weight.

Induction of active and adoptive disease. For active induction of EAE, mice were immunized subcutaneously in the flank with $100 \mu \mathrm{l}$ of CFA (Difco Laboratories, Detroit, Michigan, USA) emulsified with $200 \mu \mathrm{g}$ of $\mathrm{MOG}_{35-55}$ or $\mathrm{PLP}_{139-151}$ peptide (according to the strain of mice used) on days 0 and 7 and with 200 ng of pertussis toxin (Sigma-Aldrich, St. Louis, Missouri, USA) intraperitoneally on days $0,1,7$, and 8 . Control mice ( $n=5$ mice per group) were injected with CFA emulsified with PBS plus pertussis toxin, according to the same schedule $(3,5)$. For adoptively transferred EAE, 9 or 10 female donor C57BL/6J wild-type mice (6-8 weeks old) were primed subcutaneously with $300 \mu \mathrm{g}$ of $\mathrm{MOG}_{35-55}$ peptide in CFA distributed over four sites. After 9 or 10 days, draining lymph nodes (axillary and inguinal) and spleens were harvested, homogenized into single-cell suspension, and cultured separately in vitro in 24-well plates (Becton Dickinson Falcon, Franklin Lakes, New Jersey, USA) $\left(8.0 \times 10^{6}\right.$ cells per well $)$ with RPMI-1640 medium (Life Technologies Inc., Gaithersburg, Maryland, USA) supplemented with $10 \%$ FCS (Life Technologies Inc.), 2 $\mathrm{mM}$ L-glutamine (Life Technologies Inc.), $0.1 \mathrm{mM}$ nonessential amino acids (Life Technologies Inc.), $1 \mathrm{mM}$ sodium pyruvate (Life Technologies Inc.), $50 \mu \mathrm{M} 2$-mercapto-ethanol (Sigma-Aldrich), $100 \mathrm{U} / \mathrm{ml}$ penicillin (Life Technologies Inc.), $100 \mu \mathrm{g} / \mathrm{ml}$ streptomycin (Life Technologies Inc.), and $50 \mu \mathrm{g} / \mathrm{ml}$ of $\mathrm{MOG}_{35-55}$ peptide. After 4 days in culture and addition to medium of $2 \mathrm{U} / \mathrm{ml}$ of recombinant IL-2 (Roche Biochemicals, Monza, Italy), cells were harvested and centrifuged over Ficoll gradient (Pharmacia, Uppsala, Sweden), and $\mathrm{CD}^{+} \mathrm{T}$ cells were purified by immunomagnetic negative selection. Briefly, $\mathrm{CD}^{+} \mathrm{T}$ cells were incubated with a saturating concentration of anti-mouse CD8, B, NK, and monocyte monoclonal antibodies (Becton Dickinson PharMingen, San Diego, California, USA) for 30 minutes at $4^{\circ} \mathrm{C}$. The cells were then washed at least three times in PBS or RPMI to remove unbound antibody and then incubated with sheep anti-rat IgG-coated Dynabeads (Dynal Biotech, Oslo, Norway) at a 4:1 ratio of magnetic beads to cells for 30 minutes at $4^{\circ} \mathrm{C}$. The bead $/ \mathrm{mAb}$-coated cells were then removed by passage through a magnetic column. A second round of immunomagnetic negative selection was performed for 30 minutes at $4^{\circ} \mathrm{C}$ at a $2: 1$ ratio of magnetic beads to cells. The purity of separated cells was assessed by flow cytometry and was always at least $95 \%$. Recipient syngeneic naive female leptin-deficient or wildtype control mice received 5 Gy of irradiation before intravenous injection of $2.5 \times 10^{7}$ highly purified $\mathrm{MOG}_{35-55^{-}}$-specific CD4 ${ }^{+} \mathrm{T}$ cells in a volume of $500 \mu \mathrm{l}$ of PBS. Mice also received $200 \mathrm{ng}$ of pertussis toxin immediately after cell transfer and 1 day later. Control naive C57BL/6J ob/ob and C57BL/6J wild-type mice $(n=5$ mice per group) received 5 Gy of irradiation before injection with PBS only or $\mathrm{T}$ cells from unprimed animals plus pertussis toxin, according to the same schedule. 
Clinical and histological assessment and immunohistochemistry. Individual mice were observed daily for clinical signs of disease for up to 40 days after immunization and up to 30 days after adoptive transfer. Mice were weighed and scored blindly each day according to the clinical severity of symptoms on a scale of $0-6$, with 0.5 points for intermediate clinical findings, as follows: grade 0 , no abnormality; grade 0.5 , partial loss of tail tonicity, indicated by an inability to curl the distal end of the tail; grade 1 , reduced tail tone or slightly clumsy gait; grade 2 , tail atony, moderately clumsy gait, impaired righting ability, or any combination of these signs; grade 3 , hind-limb weakness or partial paralysis; grade 4, complete hind-limb paralysis or fore-limb weakness; grade 5, tetraplegia or moribund state; and grade 6 , death. The data were plotted as daily mean clinical scores for all animals in a particular treatment group. Scores for asymptomatic mice $($ score $=0)$ were included in the calculation of the daily mean clinical score for each group (5). The brains and spinal cords were dissected between 15 and 30 days after immunization, according to the clinical stage of disease, and fixed in 10\% formalin. Paraffin-embedded sections of $5-\mu \mathrm{m}$ thickness were cut and stained with hematoxylin and eosin (Sigma-Aldrich). Sections from 4-10 segments per mouse were examined blindly by one investigator using a published scoring system for inflammation (from $0-5)$ (19). Leptin was detected with the polyclonal antibody against leptin, A-20 (Santa Cruz Biotechonolgy Inc., Santa Cruz, California, USA), at a 1/100 dilution using an immunoperoxidase kit (Vector Laboratories, Burlingame, California, USA) according to the manufacturer's instructions and the substrate 3,3'-diaminobenzidine (DAB, $0.5 \mathrm{mg} / \mathrm{ml}$ for $4 \mathrm{~min}$ ). After the immunostaining, the sections were counterstained with hematoxylin. The intensity of the cellular staining was evaluated by an observer blinded to the experimental design according to a published scoring system (20).

\section{Figure 1}

Serum leptin increase precedes the acute onset of chronic-progressive EAE and correlates with disease susceptibility, body-weight loss, and food-intake inhibition in EAE-susceptible C57BL/6J wild-type mice but not in EAE-resistant leptin-deficient C57BL/6J ob/ob mice. (a) Mean clinical score (bars) and body weight (curves) of C57BL/6J wild-type littermate controls (black bars and triangles) and leptindeficient C57BL/6J ob/ob mice (white bars and circles) after immunization with $\mathrm{MOG}_{35-55}$ peptide. Leptin-deficient mice are EAE resistant and do not lose weight after immunization, whereas wild-type controls are EAE susceptible and lose body weight. (b) Serum leptin (bars) increases before clinical onset of EAE only in wild-type controls and is undetectable in leptin-deficient mice; this increase correlates with food-intake inhibition, which is only present in wild-type animals. (c) Simple regression analysis showing a significant correlation $(P=0.0005, r=0.89)$ between the change in serum leptin before and after immunization with $\mathrm{MOG}_{35-55}$ peptide ( $\Delta$ indicates the increase in serum leptin) and the CDI, calculated as the sum of each daily clinical score of each single mouse $(n=10)$. A significant correlation was observed in wild-type control mice but not in leptindeficient mice. One representative experiment out of two is shown. $y$, equation that defines this regression; R2, regression coefficient, $\mathrm{R}$, correlation coefficient.
Briefly, the numbers of stained cells in the parenchyma were counted for each mouse on coded sections, and section areas were measured. Data are expressed as DABstained cells per square millimeter (20).

Food intake, body weight, and leptin measurement. All groups of mice were recorded daily for food intake and body weight before and after the start of the immunization protocol for EAE induction. During this time, serum samples were obtained from tail veins of all the animals and stored at $-80^{\circ} \mathrm{C}$ for serum leptin measurement. All serum samples (dilution $1 / 20$ ) were tested in a mouse leptin-specific ELISA (R\&D Systems) according to the manufacturer's instructions; the detection limit of the assay was typically less than 22 $\mathrm{pg} / \mathrm{ml}$, and the intra- and interassay variability were $4.3 \%$ and $7.6 \%$, respectively. In vitro leptin secretion by $\mathrm{T}$ cells was measured by a mouse leptin-specific ELISA (R\&D Systems) on cell-culture supernatants derived from 72-hour cultures of highly purified $\mathrm{CD}^{+} \mathrm{T}$ cells. Cells were isolated from lymph nodes of $\mathrm{PLP}_{139-151}$ peptide immunized mice (see previous section) and stimulated in vitro with irradiated (30 Gy) syngeneic splenocytes previously pulsed with $50 \mu \mathrm{g} / \mathrm{ml} \mathrm{PLP}_{139-151}$ peptide at a responder/stimulator ratio of 1:1.
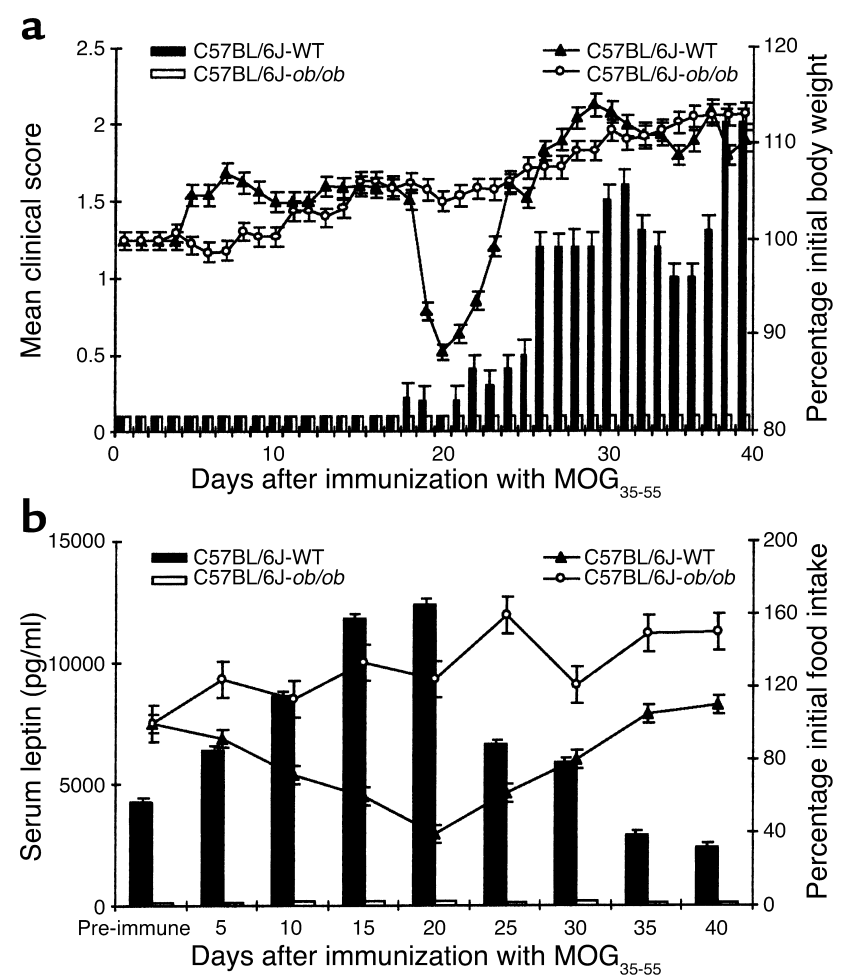

$\mathbf{C}$

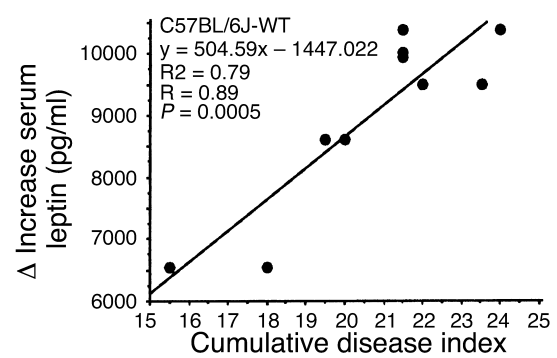



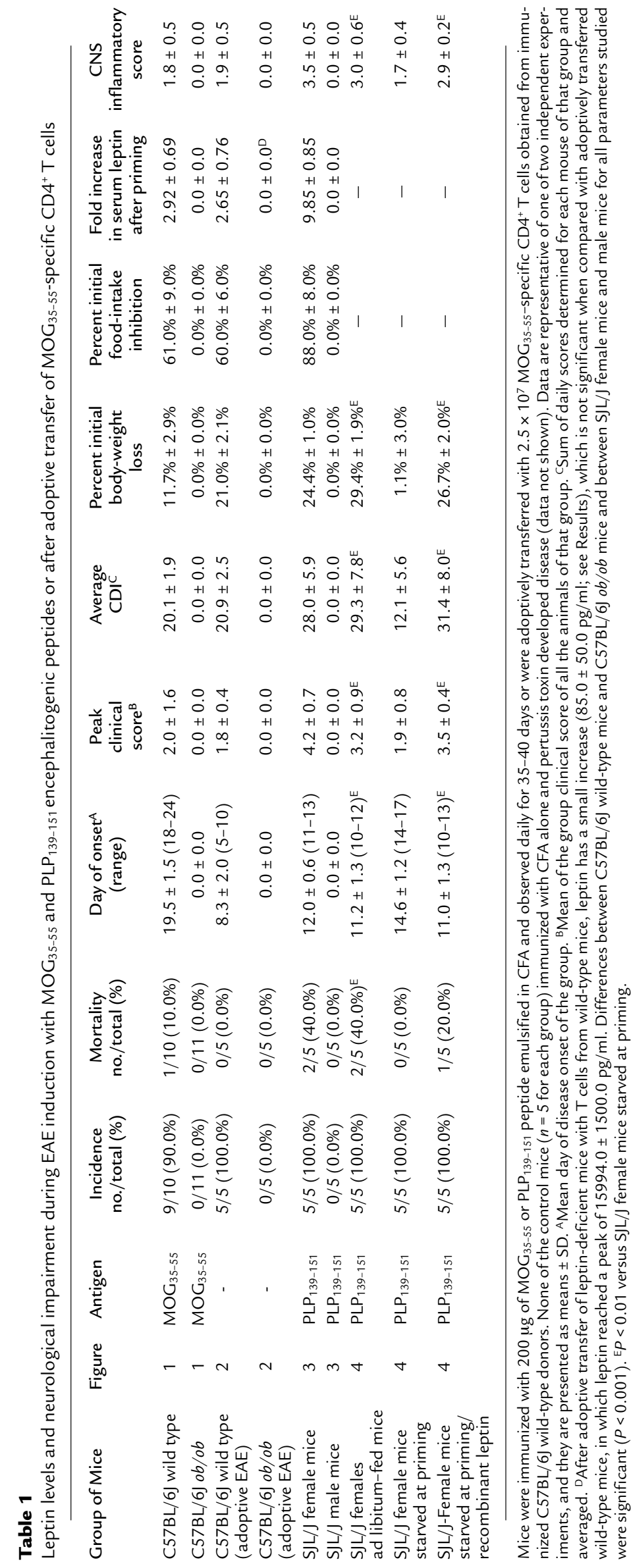

Proliferation assays and cytokine analysis. Spleen and lymph node cells were obtained from mice 15-30 days after peptide immunization, dissociated into single-cell suspensions, and cultured for proliferation assays in flat-bottom 96-well microtiter plates (Becton Dickinson Falcon) at a density of $5 \times 10^{5}$ viable cells per well in a total volume of $200 \mu \mathrm{l}$ of RPMI-1640 medium (Life Technologies Inc.) supplemented with $2 \%$ FCS (Life Technologies Inc.), 2 mM L-glutamine (Life Technologies Inc.), $0.1 \mathrm{mM}$ nonessential amino acids (Life Technologies Inc.), $1 \mathrm{mM}$ sodium pyruvate (Life Technologies Inc.), $50 \mu \mathrm{M} 2$-mercapto-ethanol (Sigma-Aldrich), $100 \mathrm{U} / \mathrm{ml}$ penicillin (Life Technologies Inc.), and 100 $\mu \mathrm{g} / \mathrm{ml}$ streptomycin (Life Technologies Inc.). Cells were cultured at $37^{\circ} \mathrm{C}$ in $100 \%$ humidity and $5 \% \mathrm{CO}_{2}$ in the presence or absence of varying concentrations of peptide (from 0-50 $\mu \mathrm{g} / \mathrm{ml})$. As a control for proliferation, antiCD3 antibody stimulation (2C11, anti-CD3ع at $10 \mathrm{ng} / \mathrm{ml}$; Becton Dickinson PharMingen) was also performed (data not shown) 48-60 hours after initiation of cell-culture supernatants $(100 \mu \mathrm{l})$ were removed from single wells and frozen at $-80^{\circ} \mathrm{C}$ for cytokine assay. IFN- $\gamma$ and IL-4 were measured by ELISA developed in our laboratory using cytokine-specific capture and detection Ab's (Becton Dickinson PharMingen) according to the manufacturer's instructions (antibodies R4-6A2 and XMG1.2 for the detection of IFN- $\gamma$ and antibodies BVD4-1D11 and BVD6-24G2 for the detection of IL-4). Standard curves for each assay were generated using recombinant mouse cytokines (IFN- $\gamma$ and IL-4, Becton Dickinson PharMingen) and the concentration of the cytokines in the cell supernatants was determined by extrapolation from the appropriate standard curve. The lower limits of detection for each assay were less than $2 \mathrm{pg} / \mathrm{ml}$ for IFN- $\gamma$ and less than 0.6 $\mathrm{pg} / \mathrm{ml}$ for IL- 4 . The remaining cells were incubated for an additional 16 hours, pulsed with $0.5 \mu \mathrm{Ci}$ per well of $\left[{ }^{3} \mathrm{H}\right]$ thymidine (AmershamPharmacia Biotech, Cologno Monzese, Milano, Italy), harvested on glass-fiber filters using a Tomtec 96-well cell harvester (Orange, Connecticut, USA), and counted in a 1205 Betaplate liquid scintillation counter (Wallac, Gaithersburg, Maryland, USA). Results are expressed as mean counts per minute \pm SD from duplicate cultures. In experiments performed with the anti-mouse leptin receptor antibody AF497 (polyclonal goat anti-mouse ObR purchased from R\&D Systems), the final concentration was $10 \mu \mathrm{g} / \mathrm{ml}$. An irrelevant polyclonal goat anti-mouse antibody was 
utilized as a control at the same final concentration (catalog no. 12061D, Becton Dickinson PharMingen). Blocking experiments were performed on lymph nodederived $\mathrm{T}$ cells from SJL/J mice immunized with $\mathrm{PLP}_{139-151}$, tested in parallel against varying concentrations of $\mathrm{PLP}_{139-151}$ peptide $(0-50 \mu \mathrm{g} / \mathrm{ml})$, concanavalin A $(5 \mu \mathrm{g} / \mathrm{ml})$, and anti-CD3E $(2 \mathrm{C} 11,10 \mathrm{ng} / \mathrm{ml})$ and in standard mixed-lymphocyte reactions (MLRs) against irradiated (30 Gy) MHC-mismatched splenocytes from C57BL/6J mice. The specificity of blocking experiments was also confirmed by adding anti-ObR Ab to spleenderived T-cell cultures obtained from leptin-receptor mutant $\mathrm{C} 57 \mathrm{BL} / \mathrm{Ks} d b / d b$ mice stimulated with concanavalin A and anti-CD3 $\varepsilon$ antibody.

Statistical analyses. Analyses were performed using the Mann-Whitney U test (for unpaired two-group analyses) and the Kruskal-Wallis ANOVA test (for analyses of three or more groups). Results are expressed as means \pm SD; $P$ values less than 0.05 were considered to be statistically significant.

\section{Results}

Serum leptin surge precedes the clinical onset of EAE and correlates with inflammatory anorexia, reduction of body weight, and EAE susceptibility. The influence of leptin in inflammatory demyelinating disease was examined using two models of EAE. A chronic-progressive model of EAE was first used to analyze the kinetics of serum leptin secretion during EAE induction in normal C57BL/6J $\left(\mathrm{H}-2^{\mathrm{b}}\right)$ wild-type mice, as compared with leptin-deficient C57BL/6J ob/ob mice immunized with $\mathrm{MOG}_{35-55}$ peptide or adoptively transferred with activated $\mathrm{MOG}_{35-55}$-specific $\mathrm{CD}^{+} \mathrm{T}$ cells. After disease induction for the appearance of clinical signs of EAE, mice were monitored for any change in body weight and food intake. Parallel serum leptin measurements were also performed. Although immunization with $\mathrm{MOG}_{35-55}$ or adoptive transfer resulted in progressive paralysis in wild-type mice, leptin-deficient C57BL/6J $o b / o b$ mice were resistant to disease induction, confirming our previous observation (11). It is interesting to note that in active-EAE-susceptible wild-type mice lost $11.7 \% \pm 2.9 \%(P<0.001)$ of their initial body weight in the early phase after immunization, before the occurrence of paralysis (Figure 1a and Table 1). Weight loss was caused by a sustained reduction $(61.0 \% \pm 9.0 \%, P<0.001)$ of daily food intake before the onset of clinical symptoms (Figure $1 \mathrm{~b}$ and Table 1$)$ and correlated with a statistically significant increase of serum leptin levels $(P<0.001)$ (Figure $1 \mathrm{~b}$ and Table 1). This increase started 3-5 days after immunization and reached a peak after 15-20 days. In contrast, body weight and food intake did not change during the observation time in leptin-deficient C57BL/6J ob/ob mice, and serum leptin remained below the detection limit of the assay (Figure 1, a and b, and Table 1). Simple regression analysis between the change in serum leptin levels before and after immunization ( $\Delta$ indicates the increase in serum leptin in Figure 1c) and the presence of disease, expressed as the cumulative disease index (CDI, calculated from the sum of the daily clinical scores), revealed a direct correlation between the degree of serum leptin increase before onset of EAE and the disease clinical score $(r=0.89, P=0.0005)$ in C57BL/6 $\mathrm{J}$ controls but not in EAE-resistant C57BL/6J ob/ob leptin-deficient mice
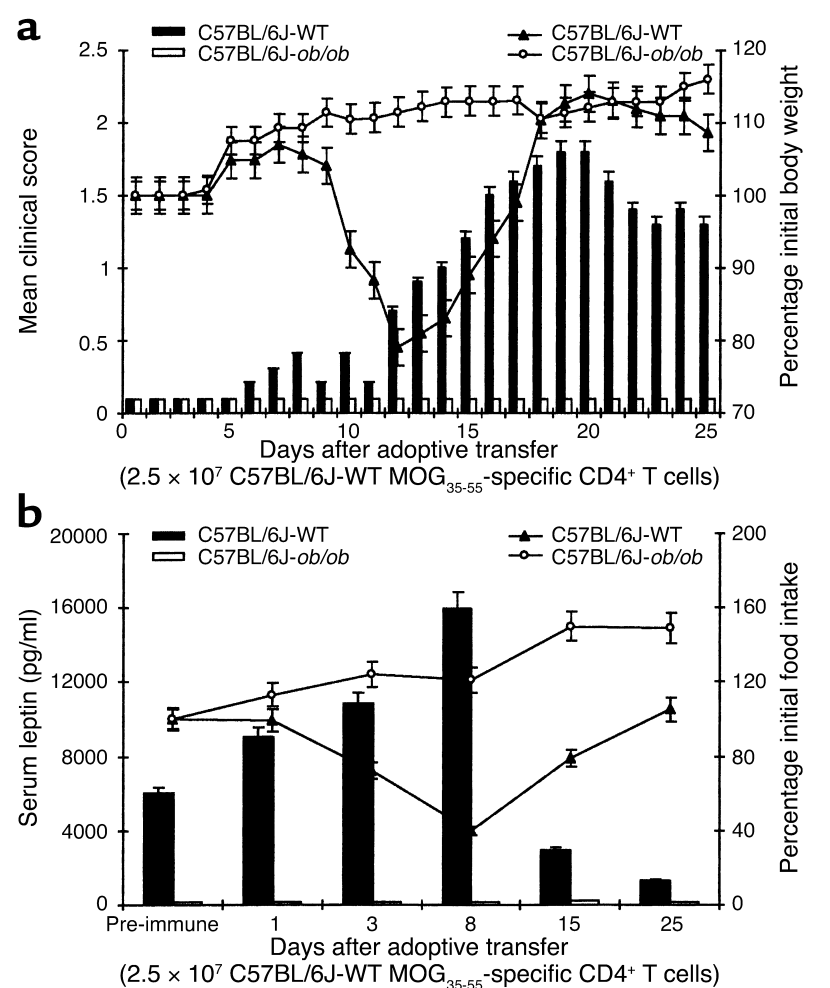

C

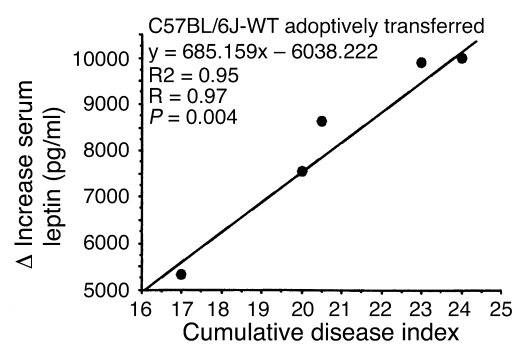

Figure 2

Serum leptin increase precedes acute onset of adoptively induced EAE and correlates with disease susceptibility, body-weight loss, and food-intake inhibition in EAE-susceptible C57BL/6J wild-type mice but not in EAE-resistant leptin-deficient C57BL/6J ob/ob mice. (a) Mean clinical score (bars) and body weight (curves) of C57BL/6J wild-type littermate controls (black bars and triangles) and leptindeficient $\mathrm{C} 57 \mathrm{BL} / 6 \mathrm{~J} o b / o b$ mice (white bars and circles) after adoptive transfer of $2.5 \times 10^{7} \mathrm{MOG}_{35-55}$-specific $\mathrm{CD}^{+} \mathrm{T}$ cells. Leptin-deficient mice are EAE resistant and do not lose weight after adoptive transfer, whereas wild-type controls are EAE susceptible and lose body weight. (b) Serum leptin (bars) significantly increases before clinical onset of EAE in wild-type controls but increases very little in leptindeficient mice; this increase correlates with food-intake inhibition, which is only present in wild-type animals. (c) Simple regression analysis showing a significant correlation $(r=0.97, P=0.004)$ between the change in serum leptin before and after adoptive trans-

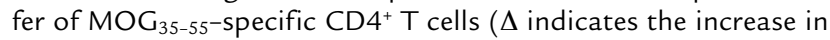
serum leptin) and the CDI, calculated as the sum of each daily clinical score of each single mouse $(n=5)$. A significant correlation was observed in wild-type control mice but not in leptin-deficient mice. One representative experiment of two is shown. 

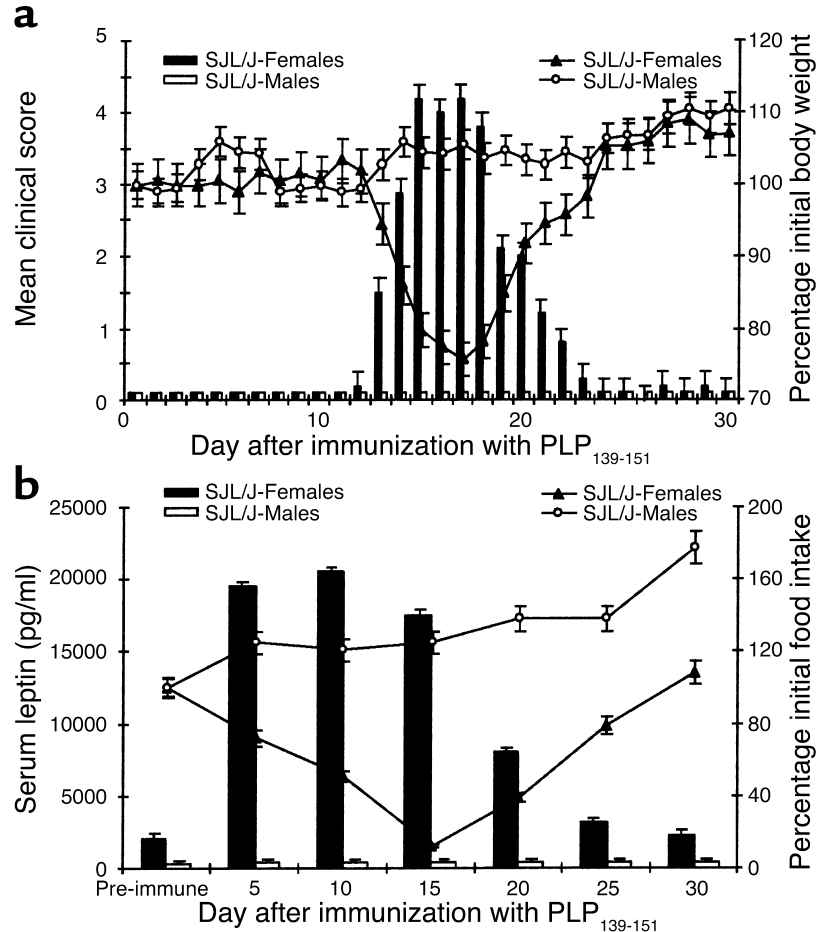

C

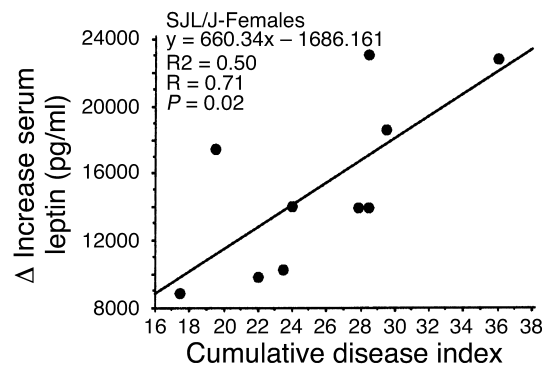

Figure 3

Serum leptin increase precedes the acute onset of relapsing-remitting EAE and correlates with disease susceptibility, body-weight loss, and food-intake inhibition in EAE-susceptible SJL/J female mice but not in EAE-resistant male mice. (a) Mean clinical score (bars) and body weight (curves) of SJL/J female mice (black bars and triangles) and male mice (white bars and circles) after immunization with PLP $139-151$ peptide. SJL/J male mice are EAE resistant and do not lose weight after immunization, whereas SJL/J female mice are EAE susceptible and lose body weight. (b) Serum leptin (bars) increases before clinical onset of EAE only in SJL/J female mice and is significantly lower in male mice in preimmune conditions $(2080.0 \pm 325.0 \mathrm{pg} / \mathrm{ml}$ in $\mathrm{SJL} / \mathrm{J}$ female mice and $470.0 \pm 100.0 \mathrm{pg} / \mathrm{ml}$ in $\mathrm{SJ} \mathrm{L} / \mathrm{J}$ male mice, $P<0.01)$; the increase correlates with food-intake inhibition present only in female mice. (c) Simple regression analysis showing a significant correlation $(r=0.71, P=0.02)$ between the difference in serum leptin before and after immunization with $\mathrm{PLP}_{139-155}$ peptide ( $\Delta$ indicates the increase in serum leptin) and the CDI, calculated as the sum of each daily clinical score of each single mouse $(n=10)$. A significant correlation was observed in $\mathrm{SJ} L /$ female mice but not in male mice. Data are accumulated and averaged from two independent experiments with similar results. $y$, equation that defines regression; $\mathrm{R} 2$, regression coefficient; $\mathrm{R}$, correlation coefficient.

(Figure 1c). In adoptively transferred wild-type mice, initial body-weight loss was $21.0 \% \pm 2.1 \%(P=0.001)$, accompanied by a reduced daily food intake $(60.0 \% \pm 6.0 \%$, $P=0.001)$ (Figure 2a and Table 1). In leptin-deficient mice, although body weight and food intake did not change significantly, serum leptin hinted at a very small increase to $85.0 \pm 50.0 \mathrm{pg} / \mathrm{ml}$, due to the wild-type transferred $T$ cells, which was not able to affect food intake and body weight. Similar to active EAE, simple regression analysis between the change in serum leptin levels before and after adoptive transfer and the appearance of the disease showed significant correlation only in wild-type animals $(r=0.97 ; P=0.004)$ (Figure 2c).

In the relapsing-remitting model of EAE induced in $\mathrm{SJL} / \mathrm{J}$ mice, in susceptible female mice the clinical symptoms appeared 10 days after immunization and resulted in ascending paralysis, preceded by a marked reduction of food intake $(88.0 \% \pm 8.0 \%, P<0.001)$ and consequent body weight $(24.4 \% \pm 1.0 \%, P<0.001)$ (Figure 3 , $a$ and $b$, and Table 1). No clinical signs and changes in food intake and body weight were noted in SJL/J male mice (Figure 3, a and b, and Table 1) (16). Increased leptin levels preceded and accompanied the acute phase of the disease only in female mice (Figure $3 \mathrm{~b}$ ), and this accounted for the reduction in food intake and body weight observed in these animals. In EAE-resistant male mice, serum leptin concentration did not show any significant fluctuation during the observation time and was also significantly lower than in female mice in basal conditions before immunization (preimmune serum leptin levels, $2080.0 \pm 325.0 \mathrm{pg} / \mathrm{ml}$ in SJL/J female mice vs. $470.0 \pm 100.0 \mathrm{pg} / \mathrm{ml}$ in SJL/J male mice; $P<0.01$ ), consistent with the gender-related dimorphism in serum leptin concentrations $(14,17)$. Similarly, simple regression analysis revealed a direct correlation between the degree of serum leptin increase before the onset of EAE and the disease clinical score $(r=0.71, P=0.02)$ (Figure 3c) in female SJL/J mice but not in EAE-resistant SJL/J male mice.

Reduction of serum leptin before the onset of EAE through acute starvation reduces disease severity and the development of pathogenic Th1 responses. Acute starvation (48 hours) in mice is known to dramatically reduce serum leptin levels (21). We addressed here whether 48 hours of starvation at priming with $\mathrm{PLP}_{139-151}$ could prevent or improve neurological symptoms in EAE-susceptible SJL/J female mice. We found that in starved mice the disease severity was significantly reduced and associated with a less marked decrease in body weight, as compared with the control ad libitum-fed group, in which neurological impairment and weight loss were observed (Figure $4 a$ and Table 1). To better characterize the immune reactivity in these animals, we next analyzed T-cell proliferation and cytokine secretion in response to $\mathrm{PLP}_{139-151}$. It is interesting to note that starved female mice showed reduced proliferation towards $\mathrm{PLP}_{139-151}$ and impaired IFN- $\gamma$ production, as compared with the ad libitum-fed control group (Figure $4, \mathrm{~b}$ and $\mathrm{c}$ ). At the same time, IL-4 secretion was increased in the starved female mice but not in ad libitum-fed animals (Figure 4d), suggesting a Th2 shift in the immune response. Furthermore, in vitro addition of exogenous leptin (rLeptin) to T-cell cultures reversed this pattern, leading to an increase in IFN- $\gamma$ production in response to 
PLP $_{139-151}$ stimulation (although at a lower level than in the ad libitum-fed control group) (Figure 4e). Similar findings were observed on administration in vivo of recombinant leptin during the 48-hour starvation period in the SJL/J female mice at priming with the encephalitogenic $\mathrm{PLP}_{139-151}$ peptide (Figure $4 \mathrm{a}$ and Table 1). Leptin replacement reversed the starvation-induced blunting of the disease course (Figure $4 \mathrm{a}$ and Table 1).

Sites of inflammation in EAE and in vitro-activated $T$ cells produce immunoreactive leptin. Given the inflammatory role of leptin, we examined its cellular expression pattern in EAE lesions and lymphoid tissues of diseased mice during active EAE. To our surprise, leptin was expressed in $\mathrm{T}$ cells and macrophages in lymph nodes (Figure 5, b and c) and acute/active inflammatory lesions in the brains and spinal cords during the acute phase of the disease and its relapse but not during remission (Figure 5, $e$ and $f, h$ and $i, k$ and $l$ ). In the brains of EAE-susceptible mice, neurons expressed increased amounts of leptin during acute/active disease as compared with neurons from control mice (Figure 5, m-o). These findings were not observed in EAEresistant leptin-deficient $\mathrm{C} 57 \mathrm{BL} / 6 \mathrm{~J} o b / o b$ or in SJL/J male mice (Figure 5, $\mathrm{d}$ and $\mathrm{m}$, and Figure 5, g and j, respectively). Similarly to actively induced disease, leptin was also expressed in inflammatory infiltrates in the brain and spinal cord of wild-type mice adoptively transferred with $\mathrm{MOG}_{35-55}-$ specific $\mathrm{CD}^{+} \mathrm{T}$ cells (Figure 5, $\mathrm{p}-\mathrm{r}$ ). On the other hand, as shown in Figure 6 ( $a$ and b), in EAE-susceptible mice the degree of leptin expression within lesions correlated with the CNS inflammatory score and therefore the severity of disease in both EAE animal models. Furthermore, we measured in vitro leptin secretion by $\mathrm{CD}^{+} \mathrm{T}$ cells derived from immunized mice and activated with PLP $139-151$ for 72 hours. Cell-culture supernatants after activation revealed the presence of leptin at a low but significant concentration when compared with unstimulated T cells $(387.5 \pm 96.01 \mathrm{pg} / \mathrm{ml}$ vs. $32.5 \pm 5.6 \mathrm{pg} / \mathrm{ml}, P=0.01$ ) (Figure 6c).

Antileptin receptor-blocking antibodies inbibit $T$ cell activation. We also tested whether blocking the leptin receptor (ObR) on $\mathrm{T}$ cells could inhibit antigen-specific and non-antigen-specific $T$ cell activation. It is interesting to note that the anti-ObR antibody (see Methods) significantly reduced the proliferative response of antigen-specific and nonantigen-specific $\mathrm{T}$ cells from SJL/J female mice (Figure 7). More specifically, addition of this antibody to the culture medium of $\mathrm{T}$ cells stimulated with PLP $_{139-151}$ resulted in a sustained reduction in proliferation, particularly at higher peptide concentrations (Figure 7a). A similar effect, but with less inhibitory activity, was also seen after exposing $\mathrm{T}$ lymphocytes to vigorous mitogenic stimuli, such as anti$\mathrm{CD} 3 \varepsilon$ antibody and concanavalin A, or to irradiated allogeneic spleen cells obtained from C57BL/6J wild-type mice in an MLR (Figure 7, b-d). Furthermore, when
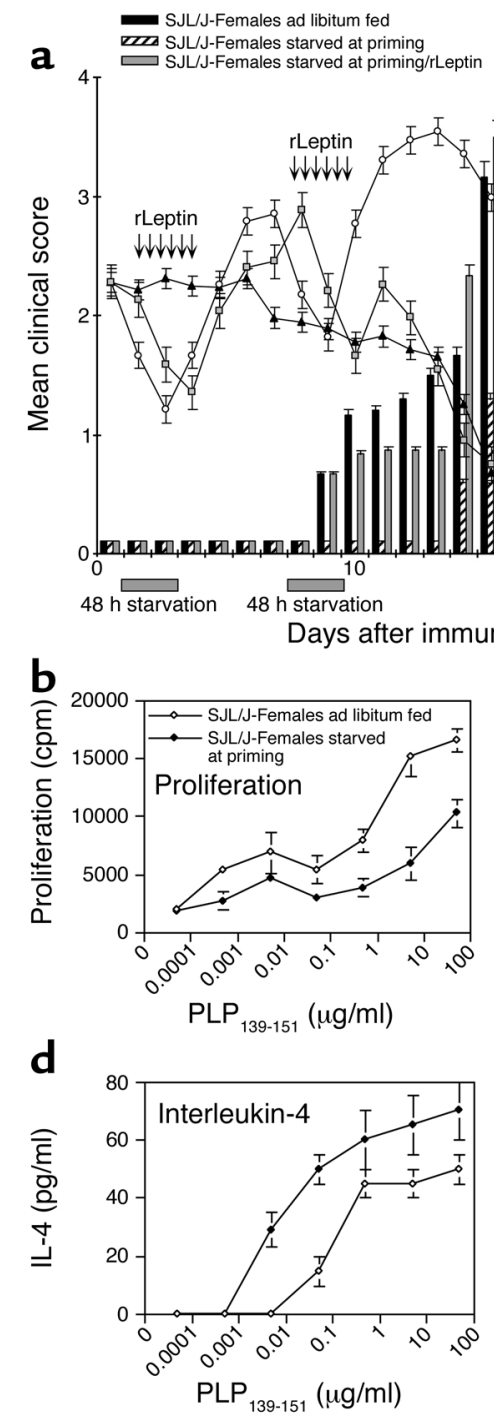

Figure 4

Starvation at priming with the encephalitogenic peptide of SJL/J females reduces the clinical severity of EAE by inducing a Th2 cytokine switch, reversible by recombinant leptin administration. (a) Mean clinical score (bars) and body weight (curves) of SJL/J female mice starved for 48 hours (hatched bars and circles), ad libitum-fed controls (black bars and triangles), and leptin-treated female mice (gray bars and squares). Starvation delayed disease onset and reduced clinical score and body-weight loss during the acute phase of the disease. Leptin replacement during the 48-hour starvation reversed the starvation-induced blunting of the disease, so that the EAE course was similar to that observed in the ad libitum-fed group. (b) Proliferative response of lymph node-derived T cells against $\mathrm{PLP}_{139-151}$ is impaired after 48 hours of starvation when compared with the control group. (c and d) Starvation for 48 hours reduced IFN- $\gamma$ secretion but increased IL-4 production. (e) Addition of recombinant leptin to T-cell cultures is able to partially restore the capacity of T cells from starved mice to secrete IFN- $\gamma$. One representative experiment out of two is shown. 


\section{Figure 5}

Lymph node and CNS expression of leptin during acute/active EAE. (a) Leptin expression in SJL/J female mouse adipose tissue used as positive control. (b and $\mathbf{c}$ ) Expression of leptin in T cells and macrophages in a draining lymph node from $\mathrm{SJL} / \mathrm{J}$ female mice after immunization with $\mathrm{PLP}_{139-151 .}$. (d) Leptin was not expressed in the brain of C57BL/6 J ob/ob mice after immunization with $\mathrm{MOG}_{35-55}$ peptide $(n=4)$. (e and $\mathbf{f})$ Expression of leptin in inflammatory infiltrates (white square) and in choroid plexus (arrow) during the acute phase of EAE in C57BL/J6 WT mice $(n=4)$. (g) Leptin was not expressed in the brain of SJL/J male mice after immunization with PLP ${ }_{139-151}$ peptide $(n=6)$. (h and i) Leptin expression in inflammatory lesions in the acute phase of EAE in SJL/J female mice $(n=6)$. (j) Cerebellum of SJL/J male mice did not express leptin after immunization with $\mathrm{PLP}_{139-151}$ peptide, whereas in $\mathbf{k}$ and $\mathbf{I}$ leptin was expressed in inflammatory infiltrates (white square) and choroid plexus (arrow) of SJL/J females. (m) Spinal cord C57BL/J6 ob/ob mice immunized with $\mathrm{MOG}_{35-55}$ peptide did not express leptin. ( $\mathbf{n}$ and $\mathbf{o}$ ) Expression of leptin in neurons (white square in $\mathbf{n}$ ) and two inflammatory infiltrates around blood vessels (arrows in $\mathbf{n}$ ) detectable during the acute phase of EAE in C57BL/6J WT mice spinal cord. ( $\mathbf{p}-\mathbf{r}$ ) Leptin expression was revealed in T cells present in inflammatory infiltrates of the brain, cerebellum, and spinal cord (arrows) of C57BL/J6 WT mice after adoptive transfer, but it was not detectable in the CNS of C57BL/6J ob/ob mice after adoptive transfer (not shown). The white squares in $\mathbf{b}, \mathbf{e}, \mathbf{h}, \mathbf{k}$, and $\mathbf{n}$ represent the zone of higher magnification shown in $\mathbf{c}, \mathbf{f}, \mathbf{i}, \mathbf{I}$, and $\mathbf{o}$, respectively. Magnifications, $\times 200(\mathbf{b}, \mathbf{d}, \mathbf{e}, \mathbf{g}, \mathbf{h}, \mathbf{j}, \mathbf{k}$, and $\mathbf{m}) ; \times 300(\mathbf{a}, \mathbf{l}$, and $\mathbf{n})$; and $\times 400(\mathbf{c}, \mathbf{f}, \mathbf{i}, \mathbf{o}$, and $\mathbf{p}-\mathbf{r})$.

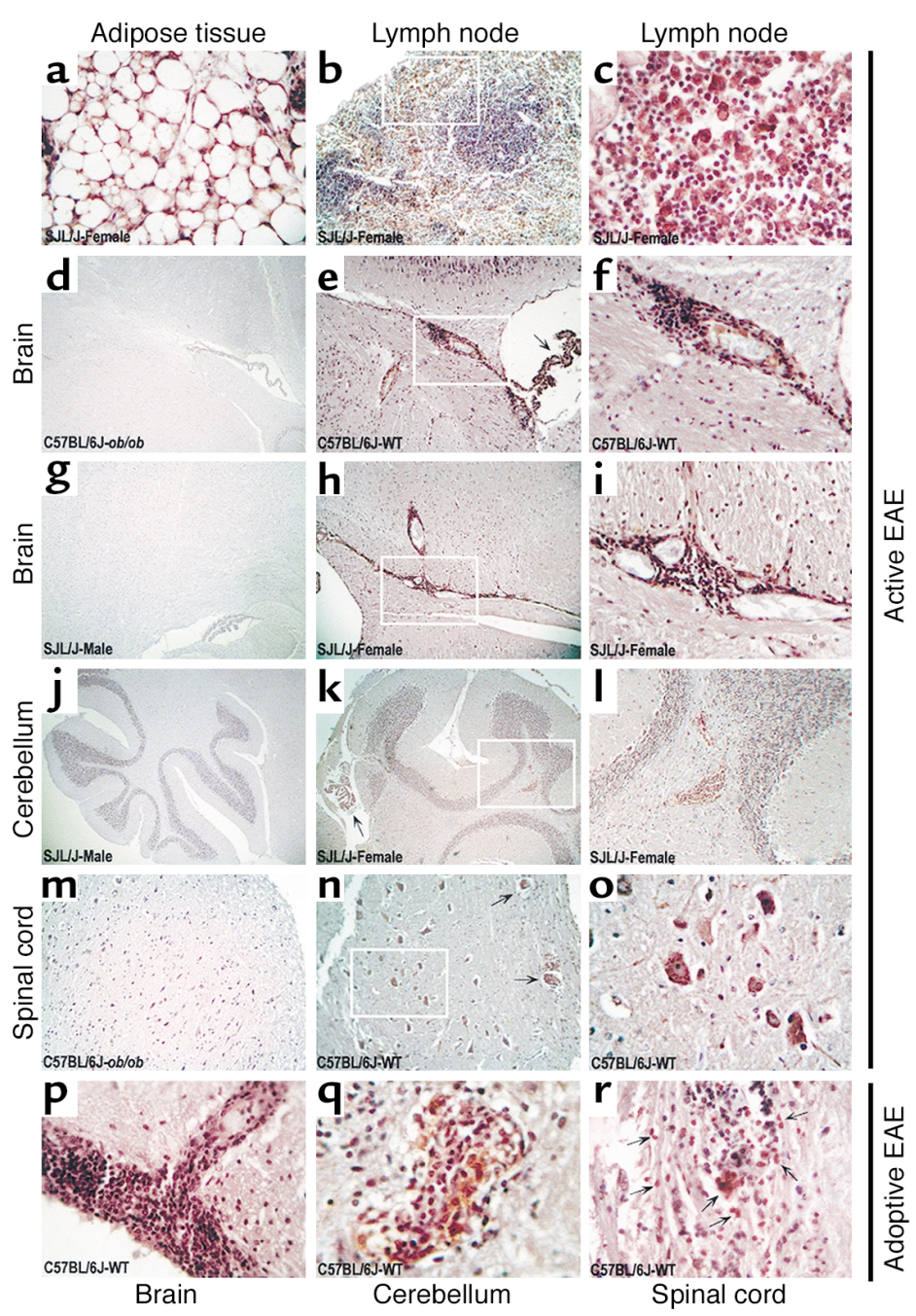

responding $\mathrm{T}$ cells were derived from $\mathrm{C} 57 \mathrm{BL} / \mathrm{Ks} d b / d b$ leptin receptor-defective mice, no significant effect of anti-ObR antibody on concanavalin A- or anti-CD3Einduced proliferation was detected (Figure 7, e and f). These effects were also observed by adding either an antileptin antibody or the fusion protein Fc:soluble leptin receptor $(\mathrm{Fc}: \mathrm{ObR})$, confirming the specificity of these results (data not shown).

\section{Discussion}

It is well corroborated that the onset of neurological symptoms after EAE induction in mice is typically preceded by a reduction in food intake and body weight (1, $5)$. The data presented here provide evidence that a significant surge in serum leptin precedes the acute phase of EAE. This increase starts after immunization with myelin antigens and anticipates the disease onset in both animal models of EAE but persists until the disease reaches its peak only in the female SJL/J relapsingremitting model (Figure 1, a and b, and Figure 2, a and b). The increased systemic secretion of leptin probably accounts for the observed food-intake inhibition and body-weight loss and directly correlates with the disease clinical score and EAE susceptibility (Figures 1c, 2c, and
$3 c)$. Indeed, acute and chronic inflammatory conditions, induced by the administration of IL- 1 , TNF- $\alpha$, and LPS, are associated with increased leptin levels, anorexia, and loss of lean body mass $(22,23)$. These findings support the view that leptin levels may be one mechanism by which anorexia is induced in acute inflammatory contexts. The lack of fluctuation of serum leptin levels after immunization could prevent disease onset and then determine a condition of EAE resistance, as observed in leptin-deficient $\mathrm{C} 57 \mathrm{BL} / 6 \mathrm{~J}$ $o b / o b$ mice and in SJL/J male mice, in which leptin levels are either undetectable or very low, respectively, as compared with those in female mice. Starvation is well known to reduce serum leptin concentrations and to impair delayed-type hypersensitivity to antigens $(9,21)$. In keeping with these findings, starvation of SJL/J female mice for 48 hours during priming with the encephalitogenic PLP $_{139-151}$ peptide reduced disease severity, impaired antigen-specific T-cell proliferation and IFN- $\gamma$ production, and led to increased IL-4 secretion (Figure 4). Since the effects of starvation can be reversed by in vitro and in vivo administration of recombinant leptin, these data support the hypothesis that leptin, together with other proinflammatory cytokines, 
a

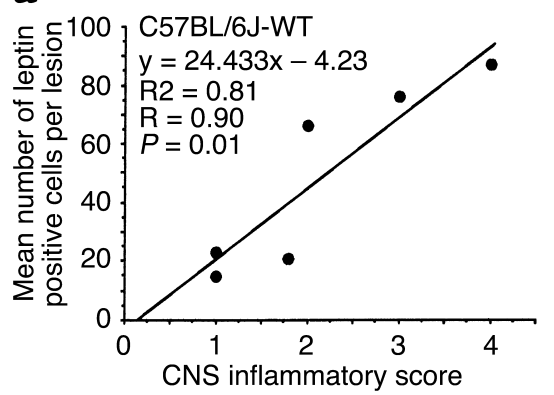

b

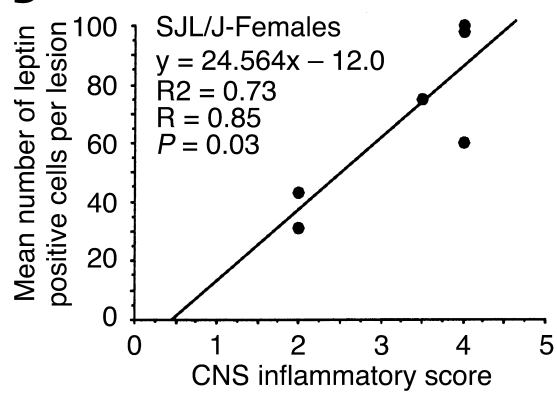

C

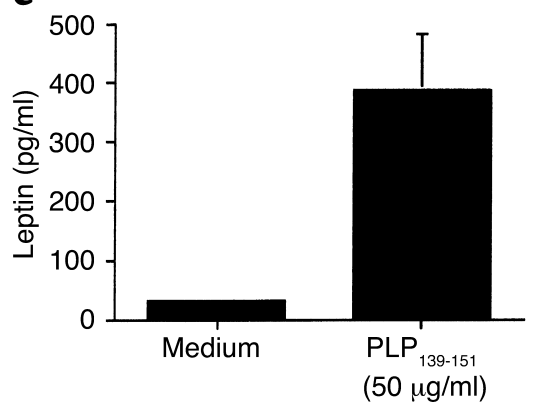

Figure 6

Simple regression analysis between the number of leptin-positive cells in active-EAE lesions and the inflammatory score in EAE-susceptible mice, and in vitro leptin secretion by activated $C D 4^{+} T$ cells. (a and $\mathbf{b}$ ) In both chronic-progressive and relapsing-remitting EAE, there is a statistically significant positive correlation between the number of leptin-positive cells in active EAE lesions and the CNS inflammatory score. (c) $\mathrm{PLP}_{139-151}$-specific CD4+ $\mathrm{T}$ cells, derived from immunized mice (see Methods), secrete on PLP ${ }_{139-151}$ 72-hour in vitro stimulation a small but consistent amount of immunoreactive leptin in the supernatants, when compared to unstimulated T cells (387.5 \pm 96.01 $\mathrm{pg} / \mathrm{ml}$ vs. $32.5 \pm 5.6 \mathrm{pg} / \mathrm{ml}$, respectively; $P=0.01)$.

may be an influential factor in the pathogenesis of demyelinating disease, favoring and sustaining Th1 pathways in the phases preceding the disease onset. The increased serum leptin secretion was accompanied by in situ production by pathogenic $\mathrm{T}$ cells and macrophages in the lymph nodes and in the acute/active demyelinating lesions of the brains and spinal cords of immunized mice (Figure 5). In situ leptin production by pathogenic Th1 lymphocytes was further confirmed by adoptive transfer experiments in EAE-susceptible mice, in which $\mathrm{CD}^{+} \mathrm{T}$ cells infiltrating the CNS stained positive for this molecule (Figure 5, p-r). In addition, leptin-deficient C57BL/6J ob/ob mice that received C57BL/6J wildtype $\mathrm{MOG}_{35-55^{-}}$specific $\mathrm{CD}^{+} \mathrm{T}$ cells had only an $85.0 \pm 50.0 \mathrm{pg} / \mathrm{ml}$ increase in serum leptin due to the wild-type transferred lymphocytes. These findings suggest that although $\mathrm{CD}^{+} \mathrm{Th} 1$ cells may contribute to leptin secretion, most of the serum leptin surge preceding the acute phase of EAE is due to adipose-tissue production. Further studies with anti-IL-1 and antiTNF- $\alpha$ blocking antibodies administered in vivo at priming with encephalitogenic peptides should address whether adipocyte-derived leptin surge in EAE may depend on these two cytokines.

\section{Figure 7}

Antileptin receptor (anti-ObR) blocking antibodies inhibit antigenspecific and non-antigen-specific T-cell activation. (a) The anti$\mathrm{PLP}_{139-151}$ proliferative response of lymph node-derived $\mathrm{T}$ lymphocytes from immunized $\mathrm{SJ} / \mathrm{J}$ female mice is completely inhibited by the addition to cell cultures of anti-ObR. (b-d) Anti-ObR partially inhibits the proliferative response of T cells from immunized SJL/J female mice toward polyclonal mitogenic stimuli such as concanavalin $\mathrm{A}$, anti-CD3 $\varepsilon$, and MLR against irradiated allogeneic splenocytes from C57BL/6J mice (see Methods). (e and f) The addition of anti-ObR antibody to concanavalin A- or anti-CD3E-activated spleen-derived $T$ cells, obtained from leptin receptor mutant $\mathrm{C} 57 \mathrm{BL} / \mathrm{Ks} d b / d b$ mice, is not able to affect their proliferative response as compared with the control antibody. One representative experiment out of four is shown.
Recently, gene-microarray analysis of Th1 lymphocytes and active MS lesions in humans revealed elevated transcripts of many genes of the neuroimmunoendocrine axis, including leptin $(24,25)$. Its transcript was abundant in the gene-expression profile of human Th1 cells, demonstrating that leptin gene expression is induced and associated with polarization toward Th1 responses that are commonly involved in T-cell-mediated autoimmune diseases such as $\operatorname{EAE}(24,25)$, as further confirmed by our in vitro leptin secretion data (Figure $6 \mathrm{c}$ ). In agreement with these observations, our results showed that antileptin receptor-blocking antibodies, as well as antileptin antibody, were able to
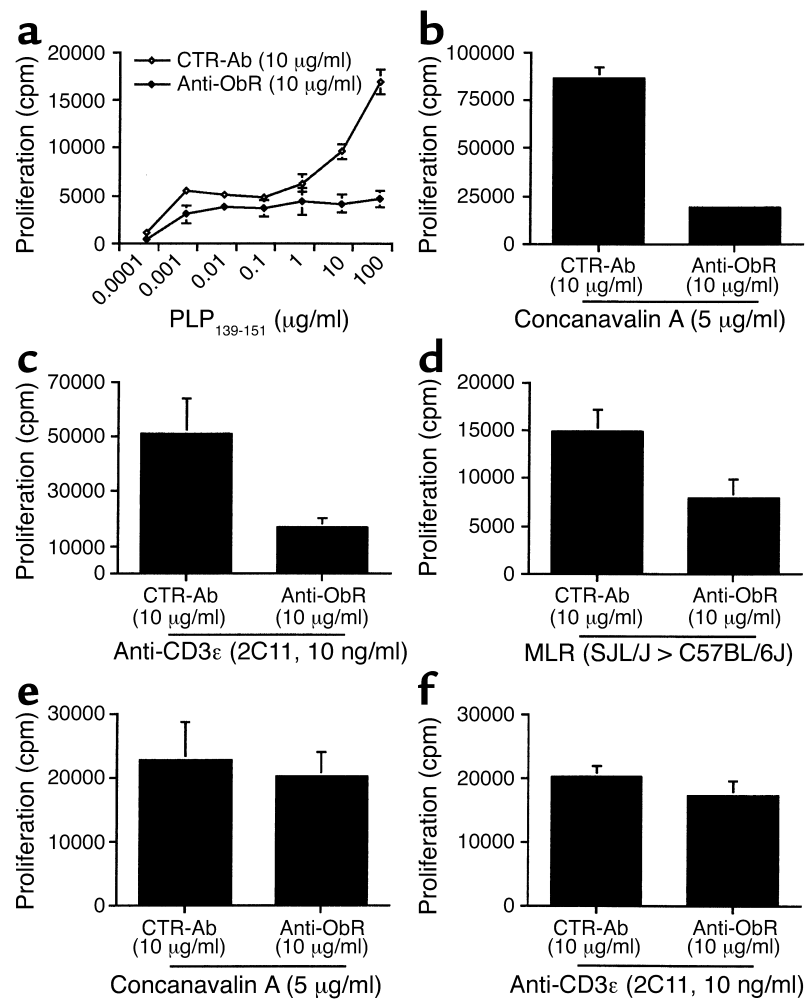

Anti-C $\overline{\mathrm{D} 3 \varepsilon(2 \mathrm{C} 11,10 \mathrm{ng} / \mathrm{ml})}$ 
reduce the proliferative response of myelin antigen-specific $\mathrm{T}$ lymphocytes, probably by interfering with an autocrine loop that may contribute to the expansion and survival of Th1 cells $(9,26)$. This effect is specific and requires leptin receptor signaling, since $\mathrm{C} 57 \mathrm{BL} / \mathrm{Ks}$ $d b / d b$ T cells are resistant to inhibition of proliferation through anti-ObR antibody (Figure 7, e and f).

Recently, it has been shown that PPAR- $\gamma$ agonists, such as 15 -deoxy- $\Delta 12,14$-prostaglandin-J2 (PGJ2) and the thiazolidinediones, decrease the duration and clinical severity of EAE by inhibiting IL-2, IL-12, IFN- $\gamma$, and TNF- $\alpha$ gene transcription in Th1 cells and macrophages (27-30). Furthermore, PPAR- $\gamma$ regulates adipocyte differentiation and suppresses leptin gene expression $(27-30)$. Based on our data, it is possible that PPAR- $\gamma$ may block EAE onset also by preventing the leptin surge, which precedes the acute phase of demyelinating disease.

An additional finding in this study was that the neurons in immunized, EAE-susceptible mice expressed an increased amount of leptin, as compared with neuronal cells from nonimmunized animals (Figure 5). These observations suggest that neurons can produce proinflammatory cytokines, influencing first the autoimmune response and then the disease severity. Paradoxically, neuronal leptin secretion, together with the production by $\mathrm{T}$ cells and macrophages in situ, could increase demyelination - since leptin is able to potentiate IFN- $\gamma$ synthesis by activated Th1 lymphocytes - and increase the expression of adhesion molecules such as ICAM-1 $(9,18)$. In addition to its immune-modulating effect, leptin has also been found to have an influence on brain development $(31,32)$. Indeed, postnatal leptin administration to $\mathrm{C} 57 \mathrm{BL} / 6 \mathrm{~J} \mathrm{ob} / \mathrm{ob}$ mice restores the appropriate brain weight and volume and the mature pattern of expression of several neuronal and glial proteins (31).

In conclusion, our results show that leptin actively contributes to the pathogenesis of EAE, influencing its onset and clinical severity. These findings suggest the possibility of an innovative therapeutic approach in the treatment of Th1 autoimmune diseases such as MS, aimed at modulating leptin concentration though initiation of an appropriate nutritional intervention and administration of drugs which interfere with the leptin pathway.

\section{Acknowledgments}

We are particularly indebted to A. Coppola for histological analysis. We also thank S. Sequino for technical advice and expert animal care in the animal facility; G. Ruggiero for ELISA reader support; P. Reynolds for reading the manuscript; and F. Perna for photographic assistance. This work was supported by grants from the Fondazione Italiana Sclerosi Multipla (FISM), Genova, Italy, and the Comitato Trenta Ore per la Vita, Mediaset, Italy. V.S. is a Consiglio Nazionale delle Ricerche Fellow, Italy.

1. Zamvil, S.S., and Steinman, L. 1990. The T lymphocyte in experimental allergic encephalomyelitis. Annu. Rev. Immunol. 8:579-621.

2. Steinman, L. 2001. Multiple sclerosis: a two-stage disease. Nat. Immunol. 2:762-764

3. Mendel, I., Kerlero de Rosbo, N., and Ben-Nun, A. 1995. A myelin oligodendrocyte glycoprotein peptide induces typical chronic experimental autoimmune encephalomyelitis in $\mathrm{H}-2^{\mathrm{b}}$ mice: fine specificity and $\mathrm{T}$ cell receptor $\mathrm{V} \beta$ expression of encephalitogenic T cells. Eur. J. Immunol. 25:1951-1959.

4. Brown, A.M., and McFarlin, D. 1981. Relapsing experimental allergic encephalomyelitis in the SJL/J mouse. Lab. Invest. 45:278-281.

5. Di Rosa, F., et al. 1998. Lack of Th2 cytokine increase during spontaneous remission of experimental allergic encephalomyelitis. Eur. J Immunol. 28:3893-3903.

6. Friedman, J.M., and Halaas, J.L. 1998. Leptin and the regulation of body weight in mammals. Nature. 395:763-770.

7. Fantuzzi, G., and Faggioni, R. 2000. Leptin in the regulation of immunity, inflammation, and hematopoiesis. J. Leukoc. Biol. 68:437-446.

8. Mancuso, P., et al. 2002. Leptin-deficient mice exhibit impaired host defense in Gram-negative pneumonia. J. Immunol. 168:4018-4024.

9. Lord, G.M., et al. 1998. Leptin modulates the T-cell mediated immune response and reverses starvation-induced immunosuppression. Nature. 394:897-901.

10. Faggioni, R., et al. 2000. Leptin-deficient $(o b / o b)$ mice are protected from T cell-mediated hepatotoxicity: role of tumor necrosis factor alpha and IL-18. Proc. Natl. Acad. Sci. USA. 97:2367-2372.

11. Matarese, G., et al. 2001. Requirement for leptin in induction and progression of experimental autoimmune encephalomyelitis. J. Immunol. 166:5909-5916

12. Whitacre, C.C. 2001. Sex differences in autoimmune disease. Nat. Immunol. 2:777-780.

13. Cua, D.J., Hinton, D.R., and Stohlman, S.A. 1995. Self-antigen-induced Th2 responses in experimental allergic encephalomyelitis (EAE)-resistant mice. Th2-mediated suppression of autoimmune disease. J. Immunol. 155:4052-4059.

14. Saad, M.F., et al. 1997. Sexual dimorphism in plasma leptin concentration. J. Clin. Endocrinol. Metab. 82:579-584.

15. Kim, S., and Voskuhl, R.R. 1999. Decreased IL-12 production underlies the decreased ability of male lymph node cells to induce experimental autoimmune encephalomyelitis. J. Immunol. 162:5561-5568.

16. Matarese, G., et al. 2001. Leptin potentiates experimental autoimmune encephalomyelitis in SJL female mice and confers susceptibility to males. Eur. J. Immunol. 31:1324-1332.

17. Matarese, G., et al. 2002. Leptin accelerates autoimmune diabetes in female NOD mice. Diabetes. 51:1356-1361.

18. Matarese, G., et al. 2002. Balancing susceptibility to infection and autoimmunity: a role for leptin? Trends Immunol. 23:182-187.

19. Bai, X.F., et al. 2000. The heat-stable antigen determines pathogenicity of self-reactive $\mathrm{T}$ cells in experimental autoimmune encephalomyelitis. J. Clin. Invest. 105:1227-1232.

20. Sabelko, K.A., Kelly, K.A., Nahm, M.H., Cross, A.H., and Russell, J.H. 1997. Fas and Fas ligand enhance the pathogenesis of experimental allergic encephalomyelitis, but are not essential for immune privilege in the central nervous system. J. Immunol. 159:3096-3099.

21. Ahima, R.S., et al. 1996. Role of leptin in the neuroendocrine response to fasting. Nature. 382:250-252.

22. Sarraf, P., et al. 1997. Multiple cytokines and acute inflammation raise mouse leptin levels: potential role in inflammatory anorexia. J. Exp. Med. 185:171-175.

23. Grunfeld, C., et al. 1996. Endotoxin and cytokines induce expression of leptin, the ob gene product, in hamsters. J. Clin. Invest. 97:2152-2157.

24. Rogge, L., et al. 2000. Transcript imaging of the development of human T helper cells using oligonucleotide arrays. Nat. Genet. 25:96-101.

25. Lock, C., et al. 2002. Gene-microarray analysis of multiple sclerosis lesions yields new targets validated in autoimmune encephalomyelitis. Nat. Med. 8:500-508.

26. Fujita, Y., et al. 2002. Leptin inhibits stress-induced apoptosis of T lymphocytes. Clin. Exp. Immunol. 128:21-26.

27. Natarajan, C., and Bright, J.J. 2002. Peroxisome proliferator-activated receptor-gamma agonists inhibit experimental allergic encephalomyelitis by blocking IL-12 production, IL-12 signaling and Th1 differentiation. Genes Immun. 3:59-70.

28. Cunard, R., et al. 2002. Regulation of cytokine expression by ligands of peroxisome proliferator-activated receptors. J. Immunol. 168:2795-2802.

29. Gosset, P., et al. 2001. Peroxisome proliferator-activated receptor- $\gamma$ activators affect the maturation of human monocyte-derived dendritic cells. Eur. J. Immunol. 31:2857-2865.

30. Niino, M., et al. 2001. Amelioration of experimental allergic encephalomyelitis by an agonist of peroxisome proliferator-activated receptor $\gamma$ (PPAR- $\gamma$ ). J. Neuroimmunol. 116:40-48.

31. Ahima, R.S., Bjorbaek, C., Osei, S., and Flier, J.S. 1999. Regulation of neuronal and glial proteins by leptin: implications for brain development. Endocrinology. 140:2755-2762.

32. Ur, E., Wilkinson, D.A., Morash, B.A., and Wilkinson, M. 2002. Leptin immunoreactivity is localized to neurons in rat brain. Neuroendocrinolo gy. 75:264-272. 